\title{
SHARP ESTIMATES FOR \\ THE NONTANGENTIAL MAXIMAL FUNCTION AND THE LUSIN AREA FUNCTION IN LIPSCHITZ DOMAINS
}

\author{
RODRIGO BAÑUELOS AND CHARLES N. MOORE
}

\begin{abstract}
Let $u$ be a harmonic function on a domain of the form $D=$ $\left\{(x, y): x \in \mathbf{R}^{n}, y \in \mathbf{R}, y>\phi(x)\right\}$ where $\phi: \mathbf{R}^{n} \rightarrow \mathbf{R}$ is a Lipschitz function. The authors show a good- $\lambda$ inequality between $A u$, the Lusin area function of $u$, and $N u$, the nontangential maximal function of $u$. This leads to an $L^{p}$ inequality of the form $\|A u\|_{p} \leq C_{p}\|N u\|_{p}$ which is sharp in the sense that $C_{p}$ is of the smallest possible order in $p$ as $p \rightarrow \infty$. For $P \in \partial D$ and $t>0$ we also consider the functions $A u(P+(0, t))$ and $N u(P+(0, t))$ and show that a corollary of the good- $\lambda$ inequality is a law of the iterated logarithm involving these two functions as $t \rightarrow 0$. If $n=1$ and $\phi$ has a small Lipschitz constant the above results are shown valid with the roles of $N u$ and $A u$ interchanged.
\end{abstract}

\section{INTRODUCTION}

In this paper we will show some good- $\lambda$ inequalities between the nontangential maximal function and the Lusin area function on Lipschitz domains. In addition, we give some applications of these, in particular, we prove a law of the iterated logarithm for the two functions which gives a precise bound on the ratios of their growth as they approach the boundary of the domain. This law of the iterated logarithm is the analogue of the result proved in [1] with the roles of the maximal function and the area function interchanged.

We call $\phi: \mathbf{R}^{n} \rightarrow \mathbf{R}$ a Lipschitz function if there exists a constant $M$ such that $|\phi(x)-\phi(y)| \leq M|x-y|$ for all $x, y \in \mathbf{R}^{n}$. The smallest such $M$ for which this inequality remains valid for all $x$ and $y$ will be called the Lipschitz constant of $\phi$. We will consider two types of Lipschitz domains, an unbounded type and a bounded type. The unbounded type will be of the form $D=\left\{(x, y): x \in \mathbf{R}^{n}, y \in \mathbf{R}, y>\phi(x)\right\}$ where $\phi$ is a Lipschitz function. The bounded domains we will consider are the starlike Lipschitz domains. These are domains $D \subseteq \mathbf{R}^{n+1}$ with the following two properties. First, we require that the boundary of $D$ is locally Lipschitz, that is, for each $P \in \partial D$ there is a coordinate system $(z, t), z \in \mathbf{R}^{n}, t \in \mathbf{R}$, and a Lipschitz function $\phi$ such that for some neighborhood $V$ of $P, V \cap D=\{(z, t): t>\phi(z)\}$. We require

Received by the editors August 14, 1987.

1980 Mathematics Subject Classification (1985 Revision). Primary 42B25, 31 A20.

The first author was supported by an NSF Postdoctoral Fellowship.

The second author was partially supported by the NSF. 
that there exists an $M$ so that all such $\phi$ have Lipschitz constant at most $M$. Second, we assume that there exists a point $P^{*} \in D$ such that for every point $P \in \partial D,\left\{t P+(1-t) P^{*}: 0 \leq t<1\right\} \subseteq D$.

For $\alpha>0$ and $h>0$ we define $\Gamma_{\alpha}=\left\{(x, y) \in \mathbf{R}^{n+1}:|x|<\alpha y\right\}$ and $\Gamma_{\alpha}^{h}=\left\{(x, y) \in \mathbf{R}^{n+1}:|x|<\alpha y, y<h\right\}$. The set $\Gamma_{\alpha}$ is an infinite cone and $\Gamma_{\alpha}^{h}$ is a truncated cone. Consider a Lipschitz graph $\left\{(x, \phi(x)): x \in \mathbf{R}^{n}\right\}$. For points $P$ in this graph, we define $\Gamma_{\alpha}(P)$ to be the cone $\Gamma_{\alpha}$ translated so that its vertex is at $P$; explicitly, if $P=(t, \phi(t))$ then $\Gamma_{\alpha}(P)=\{(x, y):|x-t|<\alpha|y-\phi(t)|\}$. We similarly define $\Gamma_{\alpha}^{h}(P)$.

Consider a function $u$ which is harmonic on an unbounded Lipschitz domain $D=\{(x, y): y>\phi(x)\}$. Consider any $\alpha>0$ for which there exists an $\alpha^{\prime}>\alpha$ such that $\Gamma_{\alpha^{\prime}}(P) \subseteq D$ for all $P \in \partial D$. (The allowable size of such $\alpha$ depends only on the Lipschitz constant of $\phi$.) We then define the nontangential maximal function and the Lusin area function of $u$ respectively by:

$$
\begin{gathered}
N_{\alpha} u(P)=\sup \left\{|u(x, y)|:(x, y) \in \Gamma_{\alpha}(P)\right\} \\
A_{\alpha} u(P)=\left(\int_{\Gamma_{\text {, }}(P)}|\nabla u(x, y)|^{2}\left(y-\phi\left(x_{0}\right)\right)^{1-n} d x d y\right)^{1 / 2}
\end{gathered}
$$

for $P=\left(x_{0}, \phi\left(x_{0}\right)\right) \in \partial D$.

For bounded starlike Lipschitz domains we consider any $\alpha>0$ for which there exists an $\alpha^{\prime}>\alpha$ such that for every $P \in \partial D$ the cone $\Gamma_{\alpha^{\prime}}^{h}(P)$ with aperture $\alpha^{\prime}$, vertical axis $\overline{P P}^{*}$ and height $h=\left|P-P^{*}\right|$ is contained in $D$. For $P \in \partial D$ consider the cone $\Gamma_{\alpha}^{h}(P)$ with aperture $\alpha$, vertical axis $\overline{P P}^{*}$ and height $h=\left|P-P^{*}\right|$. If $u$ is harmonic on $D, P \in \partial D$ we define $N_{\alpha} u(P)$ and $A_{\alpha} u(P)$ as before, except that now we use these new cones. For typographical convenience, we will always write $N_{\alpha}^{2} u(P)$ for $\left(N_{\alpha} u(P)\right)^{2}$ and $A_{\alpha}^{2} u(P)$ for $\left(A_{\alpha} u(P)\right)^{2}$.

The size of $N_{\alpha} u$ and $A_{\alpha} u$ are intimately related. Except for possibly a set of measure zero the set where they are finite is the same. (See Stein [18, pp. $201,206]$.) For $0<p<\infty$, there exists a constant $C_{p}$ such that

$$
C_{p}^{-1}\left\|A_{\alpha} u\right\|_{p} \leq\left\|N_{\alpha} u\right\|_{p} \leq C_{p}\left\|A_{\alpha} u\right\|_{p}
$$

so that the distribution functions of $N_{\alpha} u$ and $A_{\alpha} u$ have roughly the same rate of growth. Burkholder and Gundy [4] proved what are now commonly called "good- $\lambda$ inequalities" for $N_{\alpha} u$ and $A_{\alpha} u$ which give direct comparisons of their distribution functions. Later, R. Fefferman, Gundy, Silverstein and Stein [10] gave more refined versions of these. Most recently, Murai and Uchiyama [16] gave very sharp versions of these good- $\lambda$ inequalities. However, all of these authors consider harmonic functions on the upper half space $\mathbf{R}_{+}^{n+1}$. Our first goal will be to extend these results to unbounded and bounded Lipschitz domains. These results are stated in $\S 5$. One of these results, (Theorem 6), is new even for the flat case. A consequence of these will be the laws of the iterated logarithm 
stated in $\S 7$; these are also new even in the case of $\mathbf{R}_{+}^{n+1}$. To prove these good$\lambda$ inequalities the techniques we will use are fairly standard; these techniques date back to the paper of Burkholder and Gundy [4]. We consider sets where $N_{\alpha} u$ or $A_{\alpha} u$ is bounded and construct a so-called "sawtooth" domain. Our first results then involve local distribution estimates on functions which have bounded nontangential maximal functions or bounded area functions.

We call $Q \subseteq \partial D, D$ a Lipschitz domain, a cube if $Q=\left\{(x, \phi(x)): x \in Q^{\prime}\right\}$ where $Q^{\prime}$ is a cube in $\mathbf{R}^{n}$ and $\phi(x)$ is the Lipschitz function defining $D$ (either globally or locally). We will show

Theorem 1. Let $\beta>0$. Let $u$ be harmonic on an unbounded Lipschitz domain $D$ in $\mathbf{R}^{n+1}$ and suppose that for every $P \in D,|u(P)| \leq 1$. Suppose also that there exists some point $P_{0} \in \partial D$ such that $A_{\beta} u\left(P_{0}\right)<\infty$. Then for every cube $Q \subseteq \partial D$ there exists a constant $a_{Q}$ such that

$$
\frac{\sigma\left\{P \in Q:\left|A_{\beta}^{2} u(P)-a_{Q}\right|>\lambda\right\}}{\sigma(Q)} \leq c_{1} e^{-c_{2} \lambda}
$$

for every $\lambda>0$. Here $\sigma$ denotes surface measure on $\partial D$ and $c_{1}$ and $c_{2}$ are absolute constants which depend only on $\beta$, the Lipschitz constant of $D$ and the dimension $n$.

Theorem 2. Let $\alpha>\beta$. Let $u$ be harmonic on an unbounded Lipschitz domain $D$ such that $A_{\alpha} u(P) \leq 1$ for every $P \in \partial D$. Suppose that there exists a point $P_{0} \in \partial D$ such that $N_{\beta} u\left(P_{0}\right)<\infty$. Then for every cube $Q \subseteq \partial D$, there exists a constant $a_{Q}$ such that

$$
\frac{\sigma\left\{P \in Q:\left|N_{\beta} u(P)-a_{Q}\right|>\lambda\right\}}{\sigma(Q)} \leq c_{1} e^{-c_{2} \lambda}
$$

for every $\lambda>0$. Here, $c_{1}, c_{2}$ are absolute constants independent of $u$.

Theorem 1 is stronger than Theorem 2 in the sense that Theorem 1 involves $A_{\beta}^{2} u$ while Theorem 2 only estimates $N_{\beta} u$. We can, however, obtain a stronger version of Theorem 2 at least in dimension 2 with suitable restrictions on the Lipschitz constant of $\phi$ and the apertures $\alpha$ and $\beta$.

Theorem 3. Suppose $D$ is an unbounded Lipschitz domain in $\mathbf{R}^{2}$ with constant $M<1$. Suppose $1<\alpha<1 / M$ and that $\alpha>\beta$. Further suppose that $A_{\alpha} u(P) \leq 1$ for every $P \in \partial D$ and that there exists a point $P_{0} \in \partial D$ such that $N_{\beta} u\left(P_{0}\right)<\infty$. Then for every cube $Q \subseteq \partial D$, there exists a constant $a_{Q}$ such that

$$
\frac{\sigma\left\{P \in Q:\left|N_{\beta} u(P)-a_{Q}\right|>\lambda\right\}}{\sigma(Q)} \leq c_{1} e^{-c_{2} \lambda^{2}}
$$

for every $\lambda>0$. Here $c_{1}$ and $c_{2}$ are absolute constants independent of $u$.

In the case $D=\mathbf{R}_{+}^{2}$ this is essentially a theorem of Chang, Wilson and Wolff [5]. This theorem is stated in $\S 4$ since we will need to use it in the proof of Theorem 3. 
Throughout, the letters $c, C, c_{1}, c_{2}, \ldots$ indicate constants which are not necessarily the same at each occurrence. All such constants will depend only on parameters such as $\alpha, \beta$, the dimension $n$, and Lipschitz constants, but never on the harmonic function $u$.

\section{Preliminaries}

In this section we describe some results which we will need in the sequel. These are divided into two types. First, we describe some results concerning Lipschitz domains. Second, we collect some facts about BMO and functions which have subgaussian (exponential square) distributions.

Consider a bounded domain $\Omega \subseteq \mathbf{R}^{n+1}$ which is Lipschitz and starlike with respect to the point $P^{*} \in \Omega$. Suppose also that $\Omega$ has the cone condition as described previously, that is, there exists an $\alpha>0$ such that for every $P \in \partial \Omega$ the cone with aperture $\alpha$, vertex at $P$, vertical axis $\overline{P P}^{*}$ and height $\left|P-P^{*}\right|$ is completely contained in $\Omega$. For $P \in \Omega$ let $d(P)$ denote the distance from $P$ to $\partial \Omega$. Also, we let $g(P)$ denote the Green's function for $\Omega$ with pole at $P^{*}$ and let $\omega$ denote harmonic measure on $\partial \Omega$ taken with respect to the point $P^{*}$. For $P \in \Omega-\left\{P^{*}\right\}$ we let $\widehat{P}$ be the point of intersection of $\partial \Omega$ with $\left\{r\left(P-P^{*}\right)+P^{*}: r>0\right\}$ and for $P \in \partial \Omega$ we define $A(P, r)=$ $\partial \Omega \cap\left\{P^{\prime}:\left|P^{\prime}-P\right|<r\right\}$. Because of the cone condition on $\Omega$, there exists a constant $r_{0}>0$ such that $B\left(P^{*}, r_{0}\right)=\left\{P^{\prime} \in \mathbf{R}^{n+1}:\left|P^{\prime}-P\right|<r_{0}\right\} \subseteq \Omega$. In fact, there exists a constant $c(\alpha)$ depending only on $\alpha$ such that $r_{0} \geq c(\alpha) \operatorname{diam} \Omega$. Set $\Omega^{*}=\Omega-B\left(P^{*}, r_{0}\right)$. The following lemma appears in Dahlberg [6 and 7].

Lemma 1. There is a number $k>0$ such that for all $P \in \Omega^{*}$ we have

$$
k^{-1} d(P)^{n-1} g(P) \leq \omega(A(\widehat{P}, d(P))) \leq k d(P)^{n-1} g(P) .
$$

It is important to note that the number $k$ depends only on the Lipschitz constant for $\Omega$, the aperture $\alpha$, and the dimension $n$. Especially important is the fact that $k$ is independent of change of scale.

Let $\sigma$ denote surface measure on $\partial \Omega$. Another theorem of Dahlberg [6] states that $\omega$ and $\sigma$ are mutually absolutely continuous and in fact satisfy an $A^{\infty}$ condition.

Lemma 2. There exists absolute constants $b>0, a>0$ and $C>0$ such that if $E \subseteq A=A(P, r) \subseteq \partial \Omega$ then

$$
\frac{\omega(E)}{\omega(A)} \leq C\left(\frac{\sigma(E)}{\sigma(A)}\right)^{b} \text { and } \frac{\sigma(E)}{\sigma(A)} \leq C\left(\frac{\omega(E)}{\omega(A)}\right)^{a} .
$$

The constants $b, a, C$ depend only on the Lipschitz constant for $\Omega$.

The other preliminary information we need involves a few facts about BMO and functions with exponential square distributions. In [19], Strömberg gives the following alternate characterization of BMO. 
Lemma 3. Let $f: \mathbf{R}^{n} \rightarrow \mathbf{R}$ be measurable. Suppose that there exists an $s$, $0<s<\frac{1}{2}$ and $\lambda_{0}>0$ such that for every cube $Q \subseteq \mathbf{R}^{n}$ there is a constant $a_{Q}$ such that $\left|\left\{x \in Q:\left|f(x)-a_{Q}\right|>\lambda_{0}\right\}\right|<s|Q|$. Then $f \in \mathrm{BMO}$ and $\|f\|_{\mathrm{BMO}} \leq C \lambda_{0}$ where $C$ depends only on $s$.

The following two lemmas will allow us to pass from local estimates in Theorems 1,2 and 3 to global estimates required for the good- $\lambda$ inequalities in $\S 5$. The first lemma essentially appears in Murai and Uchiyama [16], although we give its proof since we require a slightly stronger result than the one obtained there.

Lemma 4. Let $f \in L_{\mathrm{loc}}^{1}\left(\mathbf{R}^{n}\right)$ such that $\|f\|_{\mathrm{BMO}} \leq 1$. Then for all $\lambda>0$, $\left|\left\{x \in \mathbf{R}^{n}:|f(x)|>2 \lambda\right\}\right|<C e^{-c \lambda}\left|\left\{x \in \mathbf{R}^{n}:|f(x)|>\lambda\right\}\right|$ where $C, c$ are positive constants which depend only on $n$.

Proof. Fix $\lambda$. We may assume that $\left|\left\{x \in \mathbf{R}^{n}:|f(x)|>\lambda\right\}\right|<\infty$. Let $\left\{Q_{i}\right\}$ be maximal dyadic cubes in $\mathbf{R}^{n}$ such that $\left|\left\{x \in Q_{i}:|f(x)|>\lambda\right\}\right|>\frac{1}{2}\left|Q_{i}\right|$. Then $\{x:|f(x)|>\lambda\} \subseteq \cup Q_{i}$ almost everywhere. Also, by the maximality of the $Q_{i}$ and the fact that $\|f\|_{\mathrm{BMO}} \leq 1$,

$$
\left|f_{Q_{i}}\right|=\left|\frac{1}{\left|Q_{i}\right|} \int_{Q_{i}} f d x\right| \leq \gamma+\lambda
$$

for every $i$, where $\gamma$ is an absolute constant. Then applying the John-Nirenberg theorem (see Garnett [11, p. 230]) to each $Q_{i}$ we obtain

$$
\begin{aligned}
\left|\left\{x \in Q_{i}:|f(x)|>2 \lambda\right\}\right| & \leq\left|\left\{x \in Q_{i}:\left|f(x)-f_{Q_{i}}\right|>\lambda-\gamma\right\}\right| \\
& \leq C e^{-c(\lambda-\gamma)}\left|Q_{i}\right|=C e^{-c \lambda}\left|Q_{i}\right| .
\end{aligned}
$$

Summing over $i$ then gives the result.

By the John-Nirenberg theorem, the condition $\|f\|_{\mathrm{BMO}} \leq 1$ is equivalent to the condition: There exists constants $C, c$ such that $C \leq C_{0}, c \geq c_{0}$, (where $C_{0}, c_{0}$ depend only on the dimension $n$ ), which have the property that for every cube $Q$ there exists a constant $a_{Q}$ such that

$$
\frac{\left|\left\{x \in Q:\left|f(x)-a_{Q}\right|>\lambda\right\}\right|}{|Q|} \leq C e^{-c \lambda} \text { for all } \lambda>0 .
$$

So Lemma 4 could have been stated with the latter condition in its hypothesis. Lemma 5 is then similar to Lemma 4; the proof is essentially the same.

Lemma 5. Let $f \in L_{\text {loc }}^{1}\left(\mathbf{R}^{n}\right)$ and suppose $f$ satisfies the condition: There exists absolute constants $c$ and $C$ such that for every cube $Q \subseteq \mathbf{R}^{n}$ there exists a constant $a_{Q}$ such that

$$
\frac{1}{|Q|}\left|\left\{x \in Q:\left|f(x)-a_{Q}\right|>\lambda\right\}\right| \leq C e^{-c \lambda^{2}} \text { for every } \lambda>0 .
$$

Then there exists constants $c_{1}$ and $C_{1}$ depending only on $c$ and $C$ such that

$$
\left|\left\{x \in \mathbf{R}^{n}:|f(x)|>2 \lambda\right\}\right| \leq C_{1} e^{-c_{1} \lambda^{2}}\left|\left\{x \in \mathbf{R}^{n}:|f(x)|>\lambda\right\}\right| .
$$




\section{THE PROOF OF THEOREM 1}

We have $\beta>0$ and $u$ harmonic on an unbounded Lipschitz domain $D$ determined by a Lipschitz function $\phi$. We also have $|u(P)| \leq 1$ on $D$. Let $Q$ be a fixed cube $Q \subseteq \partial D$. We will estimate the contribution to $A_{\beta}^{2} u(P)$, $P \in Q$, from the part of the cone $\Gamma_{\beta}(P)$ which is "close" to $Q$ and the part of the cone $\Gamma_{\beta}(P)$ which is "far" from $Q$ separately. To estimate the part which is "close" we construct an auxiliary domain $\Omega$. Choose $\gamma, \gamma^{\prime}$ such that $\gamma^{\prime}>\gamma>\beta$ and so that $\Gamma_{\gamma^{\prime}}(P) \subseteq D$ for every $P \in \partial D$. Let $S$ denote the point which is the "center" of $Q, S=\left(x_{0}, \phi\left(x_{0}\right)\right)$ where $x_{0}$ is the center of $Q^{\prime}$ and $Q=\left\{(x, \phi(x)): x \in Q^{\prime}\right\}$. There exists an $R>0$ with the following properties:

(i) Set $P^{*}=\left(x_{0}, \phi\left(x_{0}\right)+R l\left(Q^{\prime}\right)\right)$ where $l\left(Q^{\prime}\right)$ denotes the side length of $Q^{\prime}$. Then for every $P \in Q^{\prime},\left\{t P+(1-t) P^{*}: 0 \leq t<1\right\} \subseteq D$.

(ii) For every $P \in Q$ the cone $\Gamma_{\gamma}^{h}(P)$ with aperture $\gamma$, vertical axis $\overline{P P}^{*}$, vertex at $P$, and height $h=\left|P-P^{*}\right|$ is completely contained in $D$.

(iii) For every $P=(x, \phi(x)) \in Q$ the cone $\Gamma_{\beta}^{h}(P)$ with vertex at $P$, vertical axis $\left\{(x, \phi(x)+s: s>0\}\right.$, and height $h=\phi\left(x_{0}\right)+R l\left(Q^{\prime}\right)-\phi(x)$ is completely contained in the cone $\Gamma_{\gamma}^{h}(P)$ given by (ii).

We assume that $R$ is the smallest such constant for which (i), (ii) and (iii) holds for all cubes in $\partial D$. Then the constant $R$ depends only on the aperture $\beta$ and the Lipschitz constant of $\phi$.

Now set $\Omega=\bigcup_{P \in Q} \Gamma_{\gamma^{\prime}}(P) \cap\left\{(x, y): y<\phi\left(x_{0}\right)+2 R l\left(Q^{\prime}\right)\right\}$. The domain $\Omega$ is Lipschitz and starlike with respect to the point $P^{*}$. Furthermore, $\Omega$ has the property that there exists an $\varepsilon_{0}>0 \quad\left(\varepsilon_{0}\right.$ depends only on $\beta$ and the Lipschitz constant of $\phi$ ) such that for every point $P \in \partial \Omega$ there exists an $\varepsilon$ with $\gamma \geq \varepsilon \geq \varepsilon_{0}$ so that the cone $\Gamma_{\varepsilon}^{h}(P)$ with vertex at $P$, height $h=\left|P-P^{*}\right|$, aperture $\varepsilon$ and vertical axis $\overline{P P}^{*}$ is completely contained in $\Omega$. In fact, for $P \in Q \subseteq \partial \Omega$ we may take $\Gamma_{\varepsilon}^{h}(P)$ to be the cone given by (ii) above.

For $P \in Q$ set $\Gamma_{1}(P)=\Gamma_{\beta}^{h}(P)$ where the latter is the cone given by (iii) above and set $\Gamma_{2}(P)=\Gamma_{\beta}(P)-\Gamma_{1}(P)$. For $j=1,2, P \in Q, P=(x, \phi(x))$, we set

$$
A_{j} u(P)=\left(\int_{\Gamma_{j}(P)}|\nabla u(s, t)|^{2}(t-\phi(x))^{1-n} d s d t\right)^{1 / 2}
$$

so that $A_{\beta}^{2} u(P)=A_{1}^{2} u(P)+A_{2}^{2} u(P)$. We will estimate $A_{1} u$ and $A_{2} u$ separately. We first estimate $A_{2} u$.

Lemma 6. Suppose that there exists $P_{0} \in \partial D$ such that $A_{\beta} u\left(P_{0}\right)<\infty$. Then for $P_{1}$ and $P_{2}$ in $Q$,

$$
\left|A_{2}^{2} u\left(P_{1}\right)-A_{2}^{2} u\left(P_{2}\right)\right| \leq C\left|P_{1}-P_{2}\right| / l\left(Q^{\prime}\right)
$$

where $C$ is an absolute constant. 
Proof. Since $|u(P)| \leq 1$ for all $P \in D$ then there exists a constant $C$ such that $\operatorname{dist}(P, \partial D)|\nabla u(P)| \leq C$ (see Stein [18, p. 209]). So if $(s, t) \in \Gamma_{\beta}((x, \phi(x))$ then $|t-\phi(x)||\nabla u(s, t)| \leq C$. Then, setting $P_{1}=(x, \phi(x)), P_{2}=(y, \phi(y))$ we have

$$
\begin{aligned}
&\left.\left|\int_{\Gamma_{2}\left(P_{1}\right)}\right| \nabla u(s, t)\right|^{2}(t-\phi(x))^{1-n} d s d t \\
&-\int_{\Gamma_{2}\left(P_{1}\right)}|\nabla u(s, t)|^{2}(t-\phi(y))^{1-n} d s d t \mid \\
& \quad \leq \int_{\Gamma_{2}\left(P_{1}\right)}|\nabla u(s, t)|^{2} \frac{\left|(t-\phi(y))^{n-1}-(t-\phi(x))^{n-1}\right|}{(t-\phi(x))^{n-1}(t-\phi(y))^{n-1}} d s d t \\
& \quad \leq C \int_{\Gamma_{2}\left(P_{1}\right)}|\nabla u(s, t)|^{2} \frac{|\phi(x)-\phi(y)|(t-\phi(x))^{n-2}}{(t-\phi(x))^{2 n-2}} d s d t \\
& \quad \leq C\left|P_{1}-P_{2}\right| \int_{\Gamma_{2}\left(P_{1}\right)} \frac{1}{(t-\phi(x))^{n+2}} d s d t \\
& \quad \leq C\left|P_{1}-P_{2}\right| \int_{\phi(x)+c l\left(Q^{\prime}\right)}^{\infty} \frac{1}{(t-\phi(x))^{2}} d t \leq \frac{C\left|P_{1}-P_{1}\right|}{l\left(Q^{\prime}\right)} .
\end{aligned}
$$

For fixed $t$,

$$
\left|\left(\Gamma_{2}\left(P_{1}\right) \Delta \Gamma_{2}\left(P_{2}\right)\right) \cap\left\{(s, t-\phi(y)): s \in \mathbf{R}^{n}\right\}\right| \leq c(t-\phi(y))^{n-1}\left|P_{1}-P_{2}\right| .
$$

Therefore,

$$
\begin{aligned}
& \left.\left|\int_{\Gamma_{2}\left(P_{1}\right)}\right| \nabla u(s, t)\right|^{2}(t-\phi(y))^{1-n} d s d t \\
& \quad-\int_{\Gamma_{2}\left(P_{2}\right)}|\nabla u(s, t)|^{2}(t-\phi(y))^{1-n} d s d t \mid \\
& \quad=\int_{\Gamma_{2}\left(P_{1}\right) \Delta \Gamma_{2}\left(P_{2}\right)}|\nabla u(s, t)|^{2}(t-\phi(y))^{1-n} d s d t \\
& \quad \leq C \int_{\Gamma_{2}\left(P_{1}\right) \Delta \Gamma_{2}\left(P_{2}\right)} \frac{1}{(t-\phi(y))^{n+1}} d s d t \\
& \quad \leq C\left|P_{1}-P_{2}\right| \int_{\phi(y)+c l\left(Q^{\prime}\right)}^{\infty} \frac{1}{(t-\phi(y))^{2}} d t \leq \frac{C\left|P_{1}-P_{2}\right|}{l\left(Q^{\prime}\right)}
\end{aligned}
$$

Estimates (3.2) and (3.3) then give Lemma 6. Note that this estimate and the fact that $A_{\beta} u\left(P_{0}\right)<\infty$ for some $P_{0}$ imply that $A_{2} u(P)<\infty$ for all $P \in Q$.

We now estimate the contribution to $A_{\beta}^{2} u$ from $A_{1}^{2} u$. We consider the auxiliary domain $\Omega$ constructed previously and let $g$ denote the Green's function for $\Omega$ with pole at $P^{*}$ and let $\omega$ denote harmonic measure on $\partial \Omega$ with respect to $P^{*}$. The following lemma is roughly the same as Dahlberg [7, Lemma 3]. 
Lemma 7. There exists a constant $C$ independent of $Q$ such that

$$
\int_{Q} A_{1}^{2} u(P) d \omega(P) \leq C .
$$

Proof. Consider domains $\Omega_{\varepsilon} \subseteq \Omega$ such that for every $\varepsilon>0 \quad P^{*} \in \Omega_{\varepsilon}, \bar{\Omega}_{\varepsilon} \subseteq \Omega$. $\Omega_{\varepsilon}$ has smooth boundary, and $\Omega_{\varepsilon} \rightarrow \Omega$ as $\varepsilon \rightarrow 0$. (See [18, p. 206] for the construction of these.) Let $g_{\varepsilon}$ denote the Green's function for $\Omega_{\varepsilon}$ with pole at $P^{*}$. We then apply Green's theorem to the functions $(u(P))^{2}-\left(u\left(P^{*}\right)\right)^{2}$ and $g_{\varepsilon}(P)$ on $\Omega_{\varepsilon}$. Since $(u(P))^{2}-\left(u\left(P^{*}\right)\right)^{2}$ vanishes at $P=P^{*}$, standard limit arguments yield

$$
\int_{\partial \Omega_{c}}(u(P))^{2}-\left(u\left(P^{*}\right)\right)^{2} d \omega_{\varepsilon}=\iint_{\Omega_{\varepsilon}} 2|\nabla u(P)|^{2} g_{\varepsilon}(P) d P .
$$

Since $|u| \leq 1$ on $D \supseteq \Omega_{\varepsilon}$ we have

$$
\iint_{\Omega_{\varepsilon}}|\nabla u(P)|^{2} g_{\varepsilon}(P) d P \leq 1 .
$$

As $\varepsilon \rightarrow 0, \Omega_{\varepsilon} \rightarrow \Omega, g_{\varepsilon}(P) \rightarrow g(P)$ and thus we obtain

$$
\iint_{\Omega}|\nabla u(P)|^{2} g(P) d P \leq 1 .
$$

For $P \in \Omega, P^{\prime} \in Q$ set $\chi\left(P, P^{\prime}\right)=1$ if $P \in \Gamma_{1}\left(P^{\prime}\right), 0$ otherwise. Then, using Lemma 1, we obtain

$$
\begin{aligned}
1 & \geq \iint_{\Omega}|\nabla u(P)|^{2} g(P) d P \geq C \iint_{\Omega}|\nabla u(P)|^{2} d(P)^{1-n} \omega(A(\widehat{P}, d(P))) d P \\
& \geq C \iint_{\Omega}|\nabla u(P)|^{2} d(P)^{1-n} \int_{Q} \chi(P, z) d \omega(z) d P \\
& =C \int_{Q} \iint_{\Omega}|\nabla u(P)|^{2} d(P)^{1-n} \chi(P, z) d P d \omega(z) \geq C \int_{Q} A_{1}^{2} u(z) d \omega(z)
\end{aligned}
$$

which completes the proof of Lemma 7.

Applying Chebyshev's inequality to the conclusion of Lemma 7 yields

$$
\omega\left\{P \in Q: A_{1}^{2} u(P)>\lambda\right\} \leq C / \lambda \leq C \omega(Q) / \lambda
$$

the latter inequality following from Hunt-Wheeden [11, Lemma 2.1]. Since $\omega$ and $\sigma$ satisfy the $A^{\infty}$ condition (2.1) on $\partial \Omega$, then $\sigma\left\{P \in Q: A_{1}^{2} u(P)>\lambda\right\} \leq$ $C \sigma(Q) / \lambda^{b}$ for some $b>0$. Since $\phi$ is Lipschitz this implies

$$
\left|\left\{x \in Q^{\prime}: A_{1}^{2} u(x, \phi(x))>\lambda\right\}\right| \leq C\left|Q^{\prime}\right| / \lambda^{b} .
$$

Also, once again since $\phi$ is Lipschitz we can rewrite (3.1) as: For every $x$, $y \in Q^{\prime}$,

$$
\left|A_{2}^{2} u(x, \phi(x))-A_{2}^{2} u(y, \phi(y))\right|<C|x-y| / l\left(Q^{\prime}\right) \leq C_{*} .
$$

Let $x_{0}$ be the center of $Q^{\prime}$. Then combining these last two inequalities gives,

$$
\begin{aligned}
& \left|\left\{x \in Q^{\prime}:\left|A_{\beta}^{2} u(x, \phi(x))-A_{2}^{2} u\left(x_{0}, \phi\left(x_{0}\right)\right)\right|>\lambda\right\}\right| \\
& \quad \leq\left|\left\{x \in Q^{\prime}: A_{1}^{2} u(x, \phi(x))>\lambda-C_{*}\right\}\right| \leq C\left|Q^{\prime}\right| /\left(\lambda-C_{*}\right)^{b} .
\end{aligned}
$$


Choose $\lambda_{0}$ so that $C /\left(\lambda_{0}-C_{*}\right)^{b}=\frac{1}{4}$. Then Lemma 3 implies $A_{\beta}^{2} u(x, \phi(x))$ is in BMO with BMO norm bounded by a constant which depends only on the choice of $\lambda_{0}$ and hence only on the Lipschitz constant of $\phi$, the aperture $\beta$ and the dimension $n$. Thus, by the John-Nirenberg theorem, for every cube $Q^{\prime} \subseteq \mathbf{R}^{n}$,

$$
\frac{1}{\left|Q^{\prime}\right|}\left|\left\{x \in Q^{\prime}:\left|A_{\beta}^{2}(x, \phi(x))-\left(A_{\beta}^{2}(x, \phi(x))\right)_{Q^{\prime}}\right|>\lambda\right\}\right| \leq C_{1} e^{-c_{2} \lambda}
$$

for every $\lambda>0$. Since $\phi$ is Lipschitz we then obtain the result of Theorem 1 .

\section{The PROOFS OF TheOREMS 2 AND 3}

We first give a proof of Theorem 3 and then a sketch of the proof of Theorem 2 since the techniques involved in the proof of Theorem 2 are just a combination of the techniques involved in proving Theorem 2 and Theorem 3 . We first state a theorem of Chang, Wilson, and Wolff [5] which is essentially Theorem 3 in the case $D=\mathbf{R}_{+}^{2}$.

Theorem. Suppose $\alpha>0$. Then there exists constants $c_{1}$ and $c_{2}$ which depend only on $\alpha$ such that if $u$ is harmonic in $\mathbf{R}_{+}^{2}$ and $\left|A_{\alpha} u(x)\right| \leq 1$ for every $x \in \mathbf{R}$, then for every finite interval $I \subseteq \mathbf{R}$, and every $\lambda>0$,

$$
\left|\left\{x \in I:\left|u(x)-u_{I}\right|>\lambda\right\}\right| \leq c_{1} e^{-c_{2} \lambda^{2}}|I| .
$$

In Theorem 3 we seek an inequality of this form but involving $N_{\beta} u$. The proof of Theorem 3 involves techniques similar to those used in the proof of Theorem 1, and the lemma which follows will allow us to pass from information about $u$ on the boundary of the auxiliary domain $\Omega$ to information about $N_{\beta} u$ on $\partial \Omega$.

Lemma 8. Suppose $\Omega$ is a Lipschitz domain which is starlike with respect to the point $P^{*} \in \Omega$. Suppose also that $\Omega$ has an interior cone condition as previously described. Let $f$ be a function on $\partial \Omega$ which has the property that there exists constants $c_{1}$ and $c_{2}$ such that $\omega\{x \in \partial \Omega:|f(x)|>\lambda\} \leq c_{1} e^{-c_{2} \lambda^{2}}$ for every $\lambda>0$. Let $M_{\omega} f$ be the Hardy-Littlewood maximal function of $f$ taken with respect to $\omega$,

$$
M_{\omega} f(P)=\sup _{r>0} \frac{1}{\omega(A(P, r))} \int_{A(P, r)}\left|f\left(P^{\prime}\right)\right| d \omega\left(P^{\prime}\right) .
$$

Then there exists constants $c_{3}$ and $c_{4}$ depending only on $c_{1}, c_{2}$ and the Lipschitz constant of $\Omega$, such that $\omega\left\{x \in \partial \Omega: M_{\omega} f(x)>\lambda\right\} \leq c_{3} e^{-c_{4} \lambda^{2}}$ for every $\lambda>0$.

Proof. Since $\omega$ satisfies a doubling condition: if $P \in \partial \Omega$ and $r>0$ then $\omega(A(P, 2 r)) \leq c \omega(A(P, r))$ where $c$ is an absolute constant, (see HuntWheeden [12]), then $M_{\omega}$ satisfies a weak type 1 inequality

$$
\omega\left\{x \in \partial \Omega: M_{\omega} g(x)>\lambda\right\} \leq c\|g\|_{1} / \lambda
$$


for all $g \in L^{1}(\omega)$. Fix $\lambda$ and set $f=g+b$ where $g(x)=|f(x)| \chi_{\{x:|f(x)|>\lambda / 2\}}$. Then

$$
\omega\left\{M_{\omega} f>\lambda\right\} \leq \omega\left\{M_{\omega} g>\frac{\lambda}{2}\right\} \leq \frac{C\|g\|_{1}}{\lambda} \leq \frac{C}{\lambda} \int_{\lambda / 2}^{\infty} e^{-c_{2} s^{2}} d s \leq \frac{c_{3}}{\lambda^{2}} e^{-c_{4} \lambda^{2}} .
$$

Since $\omega\left\{M_{\omega} f>\lambda\right\} \leq 1$ then Lemma 8 follows by choosing (if necessary) $c_{3} \geq e^{c_{4}}$.

Proof of Theorem 3. We have $\alpha>\beta, 1<\alpha<1 / M$ where $M$ is the Lipschitz constant of $\phi$ and $\left|A_{\alpha} u(P)\right| \leq 1$ for every $P \in \partial D$. Fix a cube $Q \subseteq \partial D$, say $Q=\left\{(x, \phi(x)): x \in Q^{\prime}\right\}$ where $Q^{\prime}$ is an interval in $\mathbf{R}$. Take $\gamma, \gamma^{\prime}$ so that $\alpha>$ $\gamma^{\prime}>\gamma>\beta$ and so that $\gamma>1$. Let $x_{0}$ be the center of $Q^{\prime}$ and choose $R>0$ which has properties (i), (ii) and (iii) as in the proof of Theorem 1. Define $\Omega$ as before and set $G=\bigcup_{P \in Q} \Gamma_{\gamma^{\prime}}(P)$ so that $\Omega=G \cap\left\{(x, y): y<\phi\left(x_{0}\right)+2 R l \cdot\left(Q^{\prime}\right)\right\}$. The domain $G$ is Lipschitz, say $G$ is given by $G=\{(x, y): y>\Psi(x)\}$ where $\Psi$ is a Lipschitz function with constant $M^{\prime}<1$. Note that $\Psi(P)=\phi(P)$ for all $P \in Q$. Let $Q^{\prime \prime}$ be the cube in $\mathbf{R}$ with center $x_{0}$ and side length 3 times that of $Q^{\prime}$ and let $Q^{\prime \prime \prime}$ be the cube in $\mathbf{R}$ with center $x_{0}$ and side length 5 times that of $Q$. Set $Q_{1}=\left\{(x, \Psi(x)): x \in Q^{\prime \prime}\right\}$ and set $Q_{2}=\left\{(x, \Psi(x)): x \in Q^{\prime \prime \prime}\right\}$. We can assume $R$ is chosen large enough so that $Q_{2} \subseteq \partial \Omega$. Then on $\partial \Omega$, $Q \subseteq Q_{1} \subseteq Q_{2}$ and $Q_{2}-Q_{1}$ consists of 2 intervals in $\partial \Omega$ whose length is comparable to $l\left(Q^{\prime}\right)$. For every $P \in \partial G$, the infinite cone $\Gamma_{\gamma}(P)$ with vertical axis parallel to the $y$ axis is contained in $G$, and furthermore $\left|A_{\gamma} u(P)\right| \leq 1$ for every $P \in \partial G$. Let $H: \mathbf{R}_{+}^{2} \rightarrow G$ be a conformal map with $H(i)=P^{*}$. Then there exists a $\delta>0$ such that for every $x \in \mathbf{R}, H\left(\Gamma_{\delta}(x)\right) \subseteq \Gamma_{\gamma}(H(x))$ (see Kenig [14, Lemma 1.13]). Consider $u \circ H$. Then

$$
\int_{\Gamma_{\delta}(x)}|\nabla(u \circ H)|^{2} d A=\int_{H\left(\Gamma_{\delta}(x)\right)}|\nabla u|^{2} d A \leq \int_{\Gamma_{\digamma^{\prime}(H(x))}}|\nabla u|^{2} d A \leq 1 .
$$

Then by (4.1), there exists constants $c_{1}$ and $c_{2}$ such that

$$
\left|\left\{x \in J:\left|u \circ H(x)-(u \circ H)_{J}\right|>\lambda\right\}\right| \leq c_{1} e^{-c_{2} \lambda^{2}}|J|
$$

for every $\lambda>0$ and every interval $J \subseteq \mathbf{R}$. Since $\left|H^{\prime}(x)\right| \in A_{2}$ (see Kenig [14, Theorem 1.10]) then $\left|H^{\prime}(x)\right| \in A^{\infty}$ so there exists $b>0$ such that for every interval $I$ and $E \subseteq I$,

$$
\sigma(H(E)) / \sigma(H(I)) \leq C(|E| /|I|)^{b} .
$$

For each interval $J \subseteq \mathbf{R}$ set $a_{J}=(u \circ H)_{J}$. Then (4.2) combined with this $A^{\infty}$ condition yields: For every interval $J \subseteq \partial G$ there exists a constant $a_{J}$ such that

$$
\sigma\left\{P \in J:\left|u(P)-a_{J}\right|>\lambda\right\} \leq c_{1} e^{-c_{2} \lambda^{2}} \sigma(J) .
$$

Since $G$ was formed using cones of aperture $\gamma^{\prime}<\alpha$ then for all $P \in G$, $\operatorname{dist}(P, \partial D)|\nabla u(P)|<C$ (see Stein [18, p. 207]). In particular, for $P \in \partial \Omega-$ $Q_{1}$, 
$|\nabla u(P)| \leq C /\left|Q^{\prime}\right|$ so that if $P_{1}, P_{2} \in \partial \Omega-Q_{1}$,

$$
\left|u\left(P_{1}\right)-u\left(P_{2}\right)\right| \leq C\left|P_{1}-P_{2}\right| /\left|Q^{\prime}\right| \leq C .
$$

Let $J_{0}$ be one of the intervals which comprise $Q_{2}-Q_{1}$. Using (4.3), standard arguments (as in the case of BMO functions, see Garnett [11, p. 223]) show that $\left|a_{Q_{2}}-a_{J_{0}}\right| \leq C_{*}$ where $C_{*}$ depends only on $c_{1}, c_{2}$. By applying (4.3) to the interval $J_{0}$, we conclude that there exists a constant $C$ such that there exists a point $P_{0} \in J_{0}$ with $\left|u\left(P_{0}\right)-a_{J_{0}}\right|<C$. This combined with (4.4) shows that $\left|u(P)-a_{J_{0}}\right|<C^{\prime}$ for all $P \in \partial \Omega-Q_{1}$. Then if $\lambda>C^{\prime}$,

$$
\begin{aligned}
\sigma\{P & \left.\in \partial \Omega:\left|u(P)-a_{J_{0}}\right|>\lambda\right\}=\sigma\left\{P \in Q_{2}:\left|u(P)-a_{J_{0}}\right|>\lambda\right\} \\
& \leq \sigma\left\{P \in Q_{2}:\left|u(P)-a_{Q_{2}}\right|>\lambda-C_{*}\right\} \leq \sigma\left(Q_{2}\right) c_{1} e^{-c_{2}\left(\lambda-C_{*}\right)^{2}} \\
& =\sigma\left(Q_{2}\right) c_{3} e^{-c_{4} \lambda^{2}} \leq \sigma(\partial \Omega) c_{3} e^{-c_{4} \lambda^{2}} .
\end{aligned}
$$

This inequality remains valid for all $\lambda>0$ by taking (if necessary) $c_{3} \geq e^{c_{4}\left(C^{\prime}\right)^{2}}$. Setting $a_{\Omega}=a_{J_{0}}$, the $A^{\infty}$ condition (2.1) implies:

$$
\omega\left\{P \in \partial \Omega:\left|u(P)-a_{\Omega}\right|>\lambda\right\} \leq c_{1} e^{-c_{2} \lambda^{2}}
$$

for every $\lambda>0$, for some absolute constants $c_{1}$ and $c_{2}$.

Recall that for each $P \in \partial \Omega$ we have a cone $\Gamma_{\varepsilon}^{h}(P)$ whose vertical axis is $\overline{P P}^{*}$ and such that for $P \in Q$ we may take $\Gamma_{\varepsilon}^{h}(P) \supseteq \Gamma_{\beta}^{h}(P)$. Let $N_{1} u(P)$ be the nontangential maximal function of $u$ taken with respect to the cones $\Gamma_{\varepsilon}^{h}(P)$ if $P \in \partial \Omega-Q$ and taken with respect to the cones $\Gamma_{\beta}^{h}(P)$ if $P \in Q$. By a theorem of Hunt and Wheeden [12], $N_{1}\left(u-a_{\Omega}\right)(P) \leq M_{\omega}\left(u-a_{\Omega}\right)(P)$ for all $P \in \partial \Omega$. Combining this with (4.5) and Lemma 8 gives

$$
\omega\left\{P \in \partial \Omega: N_{1}\left(u-a_{\Omega}\right)(P)>\lambda\right\} \leq c_{1} e^{-c_{2} \lambda^{2}}
$$

for every $\lambda>0$. By the triangle inequality, $\left|N_{1} u(P)-\right| a_{\Omega}|| \leq N_{1}\left(u-a_{\Omega}\right)(P)$ and since $\omega$ and $\sigma$ satisfy the $A^{\infty}$ condition on $\partial \Omega(4.6)$ then becomes

$$
\sigma\left\{P \in \partial \Omega:\left|N_{1} u(P)-\right| a_{\Omega}||>\lambda\right\} \leq c_{1} e^{-c_{2} \lambda^{2}} \sigma(\partial \Omega) .
$$

Since $\sigma(\partial \Omega) \leq C \sigma(Q)$ we then obtain

$$
\sigma\left\{P \in Q:\left|N_{1} u(P)-\right| a_{\Omega}||>\lambda\right\} \leq c_{1} e^{-c_{2} \lambda^{2}} \sigma(Q) .
$$

This gives an estimate for the contribution to $N_{\beta} u(P), P \in Q$ from points "close by $Q$." We now prove an estimate like Lemma 6 to control the contribution to $N_{\beta} u$ from points "far away" from $Q$.

Lemma 9. Suppose that there exists a point $P_{0} \in \partial D$ such that $N_{\beta} u\left(P_{0}\right)<\infty$. For $P \in Q$ set

$$
N_{2} u(x)=\sup \left\{|u(s, t)|:(s, t) \in \Gamma_{\beta}(P), t>\phi\left(x_{0}\right)+R l\left(Q^{\prime}\right)\right\} .
$$


Then for all $P_{1}, P_{2} \in Q$,

$$
\left|N_{2} u\left(P_{1}\right)-N_{2} u\left(P_{2}\right)\right| \leq C\left|P_{1}-P_{2}\right| / l\left(Q^{\prime}\right)
$$

where $C$ is an absolute constant.

Proof. Say $P_{1}=(x, \phi(x)), P_{2}=(y, \phi(y))$. Note that $|x-y| \approx\left|P_{1}-P_{2}\right|$ since $\phi$ is Lipschitz. Suppose $(s, t) \in \Gamma_{\beta}\left(P_{1}\right)$ with $t>\phi\left(x_{0}\right)+R l\left(Q^{\prime}\right)$. Then the point $(s+y-x, t) \in \Gamma_{\beta}\left(P_{2}\right)$. Once again, since $\operatorname{dist}(P, \partial D)|\nabla u(P)| \leq C$ for $P \in G$, then for $P \in G \cap\left\{(w, z): z>\phi\left(x_{0}\right)+R l\left(Q^{\prime}\right)\right\},|\nabla u(P)|<C /\left|Q^{\prime}\right|$. Therefore,

$$
|u(s, t)-u(s+y-x, t)| \leq \frac{C|x-y|}{l\left(Q^{\prime}\right)} \leq \frac{C\left|P_{1}-P_{2}\right|}{l\left(Q^{\prime}\right)} .
$$

Thus, $N_{2} u\left(P_{1}\right) \leq C\left|P_{1}-P_{2}\right| / l\left(Q^{\prime}\right)+N_{2} u\left(P_{2}\right)$. Similarly we obtain such an inequality with $P_{1}$ and $P_{2}$ interchanged to obtain the estimate (4.8). Note that this result and the fact that $N_{\beta} u\left(P_{0}\right)<\infty$ implies that $N_{2} u(P)<\infty$ for all $P \in Q$.

Now set $b_{Q}=N_{2} u\left(x_{0}, \phi\left(x_{0}\right)\right)$. Then we have the estimate

$$
\sigma\left\{P \in Q:\left|N_{2} u(P)-b_{Q}\right|>\lambda\right\} \leq c_{1} e^{-c_{2} \lambda^{2}} \sigma(Q)
$$

for every $\lambda>0$ which follows trivially by taking $c_{1}$ large enough. Using the fact that for any set $E \subseteq \mathbf{R},|E| \approx \sigma\{(x, \phi(x)): x \in E\}$ and the estimates (4.7) and (4.9), Theorem 3 will follow from the following lemma.

Lemma 10. Let $f$ and $g$ be functions defined on a cube $Q \subseteq \mathbf{R}^{n}$. Suppose that there exists constants $a_{Q}, b_{Q}, c_{1}$ and $c_{2}$ such that

$$
\left|\left\{x \in Q:\left|f(x)-a_{Q}\right|>\lambda\right\}\right| \leq c_{1} e^{-c_{2} \lambda^{2}}|Q|
$$

and

$$
\left|\left\{x \in Q:\left|g(x)-b_{Q}\right|>\lambda\right\}\right| \leq c_{1} e^{-c_{2} \lambda^{2}}|Q|
$$

for every $\lambda>0$. Then for every $\lambda>0$,

$$
\left|\left\{x \in Q:\left|\max (f(x), g(x))-\max \left(a_{Q}, b_{Q}\right)\right|>\lambda\right\}\right| \leq 2 c_{1} e^{-c_{2} \lambda^{2}}|Q| .
$$

Proof. We can assume $a_{Q} \geq b_{Q}$. Then

$$
\begin{aligned}
\{x \in Q: & \left.\left|\max (f(x), g(x))-a_{Q}\right|>\lambda\right\} \\
= & \left\{x \in Q: f(x) \geq g(x),\left|f(x)-a_{Q}\right|>\lambda\right\} \\
& \cup\left\{x \in Q: g(x) \geq f(x),\left|g(x)-a_{Q}\right|>\lambda\right\} \\
\subseteq & \left\{x \in Q:\left|f(x)-a_{Q}\right|>\lambda\right\} \cup\left\{x \in Q: g(x) \geq f(x), g(x)-a_{Q}>\lambda\right\} \\
& \cup\left\{x \in Q: g(x) \geq f(x), g(x)-a_{Q}<-\lambda\right\} \subseteq\left\{x \in Q:\left|f(x)-a_{Q}\right|>\lambda\right\} \\
& \cup\left\{x \in Q: g(x) \geq f(x), g(x)-b_{Q}>\lambda\right\} \\
& \cup\left\{x \in Q: g(x) \geq f(x), f(x)-a_{Q}<-\lambda\right\} \\
\subseteq & \left\{x \in Q:\left|f(x)-a_{Q}\right|>\lambda\right\} \cup\left\{x \in Q:\left|g(x)-b_{Q}\right|>\lambda\right\}
\end{aligned}
$$

and the result follows. 
We now give a short sketch of the proof of Theorem 2. Fix a cube $Q$ and form the auxiliary domain $\Omega$ as in the proof of Theorem 3. As before, let $g(P)$ denote the Green's function for $\Omega$ with pole at $P^{*}$ and let $\omega$ denote harmonic measure on $\partial \Omega$ with respect to the point $P^{*}$. Green's theorem yields

$$
\int_{\partial \Omega}\left|u(P)-u\left(P^{*}\right)\right|^{2} d \omega(P)=\iint_{\Omega} 2|\nabla u(P)|^{2} g(P) d P .
$$

The inequalities in Lemma 7 can all be reversed so that we then have

$$
\int_{\partial \Omega}\left|u(P)-u\left(P^{*}\right)\right|^{2} d \omega(P) \leq C .
$$

Using the same procedure as in the proof of Theorem 3, we can turn this into an estimate involving the nontangential maximal function: There exists a constant $a_{Q}$ such that

$$
\sigma\left\{P \in Q:\left|N_{1} u(P)-a_{Q}\right|>\lambda\right\} \leq C \sigma(Q) / \lambda^{b}
$$

for some $b>0, C>0$ independent of $Q$. Also, by Lemma 9, a similar estimate holds for $\mathrm{N}_{2} u$ on $Q$ :

$$
\sigma\left\{P \in Q:\left|N_{2} u(x)-b_{Q}\right|>\lambda\right\}<C \sigma(Q) / \lambda^{b}
$$

which follows trivially by taking $C$ large enough. Then the proof of Lemma 10 shows that we get such an estimate for $N_{\beta} u$ : For every $Q \subseteq \partial D$ there exists a constant $c_{Q}$ such that

$$
\sigma\left\{P \in Q:\left|N_{\beta} u(P)-c_{Q}\right|>\lambda\right\} \leq C \sigma(Q) / \lambda^{b} .
$$

The theorem then follows from Lemma 3.

The main difference between Theorems 1 and 2 is the presence of the squared term $A_{\beta}^{2} u$ in Theorem 1 , while in Theorem 2 we merely obtain an estimate involving $N_{\beta} u$. The difference occurs in (4.10). In Theorem 1 we assume that the left-hand side of $(4.10)$ is bounded which gives an estimate on the righthand side which is essentially the square of the area function. In Theorem 2 , we assume that the right-hand side of (4.10) is bounded, and this only gives an estimate involving $N_{\beta} u$ on the left-hand side.

In terms of the applications which follow, a higher-dimensional version of Theorem 3 would be a stronger theorem than Theorem 2 . This would be attainable if one could show a result like the theorem of Chang, Wilson and Wolff where the function $u$ was only assumed to satisfy $L u=0$ for some strongly elliptic operator $L$. Then one could replace the conformal map $H$ in the proof of Theorem 3 by $H^{*}: \mathbf{R}_{+}^{n} \rightarrow D$ given by $H^{*}(s, t)=(s, t+\phi(s))$ and a computation then shows that $L\left(u \circ H^{*}\right)=0$ for some strongly elliptic operator $L$. The rest of the proof would be exactly the same as in Theorem 3 .

\section{GOOD- $\lambda$ INEQUALITIES FOR $N u$ AND $A u$ ON LIPSCHITZ DOMAINS}

Theorems 1, 2, 3 will lead almost immediately to the good- $\lambda$ inequalities in Theorems 4, 5, 6 below. In each of Theorems 4, 5, 6 we let $u$ be a harmonic 
function on an unbounded Lipschitz domain $D=\{(x, y): y>\phi(x)\}$ and we let $\alpha>\beta$.

Theorem 4. There exists a constant $K>1$ and constants $c_{1}$ and $c_{2}$ such that if $\varepsilon>0, \lambda>0$ then

$$
\sigma\left\{P \in \partial D: A_{\beta} u(P)>K \lambda, N_{\alpha} u(P)<\varepsilon \lambda\right\} \leq c_{1} e^{-c_{2} / \varepsilon^{2}} \sigma\left\{P \in \partial D: A_{\beta} u(P)>\lambda\right\} .
$$

Theorem 5. There exists constants $c_{1}$ and $c_{2}$ such that if $\varepsilon>0$ and $\lambda>0$ then

$$
\sigma\left\{P \in \partial D: N_{\beta} u(P)>2 \lambda, A_{\alpha} u(P)<\varepsilon \lambda\right\} \leq c_{1} e^{-c_{2} / \varepsilon} \sigma\left\{P \in \partial D: N_{\beta} u(P)>\lambda\right\} .
$$

Theorem 6. Assume now that $D \subseteq \mathbf{R}^{2}$ with Lipschitz constant $M>1$ and $1<\alpha<1 / M$ and $\alpha>\beta$. Then there exists constants $c_{1}$ and $c_{2}$ such that if $\varepsilon>0, \lambda>0$ then

$$
\sigma\left\{P \in \partial D: N_{\beta} u(P)>2 \lambda, A_{\alpha} u(P)<\varepsilon \lambda\right\} \leq c_{1} e^{-c_{2} / \varepsilon^{2}} \sigma\left\{P \in \partial D: N_{\beta} u(P)>\lambda\right\} .
$$

Theorems 4 and 5 are the analogue for Lipschitz domains of the results of Murai and Uchiyama [16]. Weaker forms of these theorems were proved by Dahlberg [7]. However, Theorem 6 is new even in the case $D=\mathbf{R}_{+}^{2}$. The corresponding result for martingales was first proved by Burkholder [3], (see also Corollary 3.1 in [5]). As it is often the case with these type of results, our proof is modeled after the martingale proof.

Proof of Theorem 4. Fix $\varepsilon$ and $\lambda$; we may assume that $\sigma\left\{A_{\beta} u>\lambda\right\}$ is finite. Let $E=\left\{P \in \partial D: N_{\alpha} u(P)>\varepsilon \lambda\right\}$ and set $W=\bigcup_{P \in E^{c}} \Gamma_{\alpha}(P)$; we may also assume that $E^{c}$ is nonempty so that $W$ exists. Then $W$ is a subdomain of $D$ and in fact $W$ is a Lipschitz domain with constant determined by $\alpha$. Say that $W=\{(x, \Psi(x)): y>\Psi(x)\}$ where $\Psi$ is a Lipschitz function. On $W$, $|u| \leq \varepsilon \lambda$ so by Theorem 1 , for every cube $Q \subseteq \partial W$ there exists a constant $a_{Q}$ such that

$$
\sigma\left\{P \in Q:\left|A_{\beta}^{2} u(P)-a_{Q}\right|>\eta\right\} \leq c_{1} e^{-c_{2} \eta / \varepsilon^{2} \lambda^{2}} \sigma(Q)
$$

for every $\eta>0$. (Here $\sigma$ represents surface measure on $\partial W$.) Since $|F| \approx$ $\sigma\{(x, \Psi(x)): x \in F\}$ for any $F \subseteq \mathbf{R}^{n}$, it follows that $\left\|A_{\beta}^{2} u(x, \Psi(x))\right\|_{\mathrm{BMO}} \leq$ $C \varepsilon^{2} \lambda^{2}$. Then by Lemma 4 , for all $\eta>0$,

$$
\left|\left\{x \in \mathbf{R}^{n}: A_{\beta}^{2} u(x, \Psi(x))>2 \eta\right\}\right| \leq C_{1} e^{-c_{2} \eta / \varepsilon^{2} \lambda^{2}}\left|\left\{x \in \mathbf{R}^{n}: A_{\beta}^{2} u(x, \Psi(x))>\eta\right\}\right|
$$

choosing $\eta=\lambda^{2}$ yields

$$
\begin{aligned}
\mid\{x & \left.\in \mathbf{R}^{n}: A_{\beta} u(x, \Psi(x))>2 \lambda\right\} \mid \\
& \leq c_{1} e^{-c_{2} / \varepsilon^{2}}\left|\left\{x \in \mathbf{R}^{n}: A_{\beta} u(x, \Psi(x))>\lambda\right\}\right| .
\end{aligned}
$$

We now need a lemma whose purpose will soon become apparent. 
Lemma 11. Let $u$ be a harmonic function on a cone $\Gamma_{\gamma}(x, 0), x \in \mathbf{R}^{n}$, and let $d>0$. Then there exists an absolute constant $L$ depending only on $\gamma$ such that

$$
\int_{\Gamma_{;}(x, d)}|\nabla u(s, t)|^{2}(t-d)^{1-n} d s d t \leq L \int_{\Gamma_{y}(x, 0)}|\nabla u(s, t)|^{2} t^{1-n} d s d t .
$$

We remark that this is obviously true with $L=1$ when $n=1$.

Proof. For $(s, t) \in \Gamma_{y}(x, d)$ let $B(s, t)$ be a ball with center $(s, t)$ and radius $r=\varepsilon d$ where $\varepsilon$ is chosen so that $B(s, t) \subseteq \Gamma_{\gamma}(x, 0)$ for all such $(s, t)$. Note that $\varepsilon$ depends only on $\gamma$. Then

$$
\begin{aligned}
& \int_{\Gamma_{i}(x, d)}|\nabla u(s, t)|^{2}(t-d)^{1-n} d s d t \\
& \quad \leq \int_{\Gamma_{i}(x, d)} \frac{1}{|B(s, t)|} \int_{B(s, t)}|\nabla u(w, z)|^{2} d w d z(t-d)^{1-n} d s d t \\
& \quad \leq \frac{c}{d^{n+1}} \int_{\Gamma_{;}(x, 0)} \int_{\Gamma_{;}(x, d)} \chi_{B(w, z)}(s, t)(t-d)^{1-n} d s d t|\nabla u(w, z)|^{2} d w d z .
\end{aligned}
$$

We estimate the inside integral.

For $z>3 d$,

$$
\int_{\Gamma_{;}(x, d)} \chi_{B(w, z)}(s, t)(t-d)^{1-n} d s d t \leq c z^{1-n}|B(w, z)| \leq c d^{n+1} z^{1-n}
$$

For $z \leq 3 d$,

$$
\begin{aligned}
& \int_{\Gamma_{i}(x, d)} \chi_{B(w, z)}(s, t)(t-d)^{1-n} d s d t \\
& \quad \leq \int_{\Gamma_{\gamma}(x, d) \cap\{(s, t): t<(3+\varepsilon) d\}}(t-d)^{1-n} d s d t \\
& \quad \leq c \int_{0}^{(2+\varepsilon) d} \int_{|s|<\gamma t} t^{1-n} d s d t \\
& \quad=c d^{2} \leq c d^{n+1} z^{1-n} .
\end{aligned}
$$

Combining (5.2), (5.3) and (5.4) gives the result of the lemma.

We can now complete the proof of Theorem 4 . Choose $K=2 \sqrt{L}$ where $L$ is the constant from Lemma 11 corresponding to the aperture $\beta$. Then using (5.1) and Lemma 11 we have,

$$
\begin{aligned}
\sigma\{P & \left.\in \partial D: A_{\beta} u(P)>K \lambda, N_{\alpha} u(P)<\varepsilon \lambda\right\}=\sigma\left\{P \in E^{c}: A_{\beta} u(P)>K \lambda\right\} \\
& \leq \sigma\left\{P \in \partial W: A_{\beta} u(P)>K \lambda\right\} \leq C\left|\left\{x \in \mathbf{R}^{n}: A_{\beta} u(x, \Psi(x))>K \lambda\right\}\right| \\
& \leq c_{1} e^{-c_{2} / \varepsilon^{2}}\left|\left\{x \in \mathbf{R}^{n}: A_{\beta} u(x, \Psi(x))>K \lambda / 2\right\}\right| \\
& \leq c_{1} e^{-c_{2} / \varepsilon^{2}}\left|\left\{x \in \mathbf{R}^{n}: A_{\beta} u(x, \phi(x))>K \lambda / 2 \sqrt{L}\right\}\right| \\
& \leq c_{1} e^{-c_{2} / \varepsilon^{2}} \sigma\left\{P \in \partial D: A_{\beta} u(P)>\lambda\right\} .
\end{aligned}
$$


Proofs of Theorems 5 and 6 . The proofs of these are almost the same so we will do them simultaneously. The proof is very similar to the proof of Theorem 4. Fix $\varepsilon$ and $\lambda$; we may assume that $\sigma\left\{N_{\beta} u>\lambda\right\}$ is finite. Let $E=\{P \in$ $\left.\partial D: A_{\alpha} u>\varepsilon \lambda\right\}$ and we may also assume that $E^{c}$ is nonempty. Pick $\gamma$ so that $\alpha>\gamma>\beta$; in the case of Theorem 6 pick $\gamma$ so that $\gamma>1$. Now set $W=\bigcup_{P \in E^{c}} \Gamma_{\gamma}(P)$. Then $W$ is a subdomain of $D$ and in fact $W$ is a Lipschitz domain with constant determined by $\gamma$. Say $W=\{(x, y): y>\Psi(x)\}$. For $P \in \partial W \cap \partial D=E^{c}=\{(x, y): y=\Psi(x)=\phi(x)\}$ it is clear that $A_{\gamma} u(P) \leq$ $A_{\alpha} u(P)<\varepsilon \lambda$. If $P \in \partial W-\partial D$, then there exists a point $P^{\prime} \in \partial W \cap \partial D$ such that $P \in \overline{\Gamma_{\gamma}\left(P^{\prime}\right)} \subseteq \Gamma_{\alpha}\left(P^{\prime}\right)$. A slight variation of Lemma 11 then shows that $A_{\gamma} u(P) \leq L \varepsilon \lambda$ where $L$ is a constant depending only $\gamma, \alpha$ and the dimension $n$. In the case of Theorem 5 the proof proceeds as in the proof of Theorem 4; Theorem 2 combined with Lemma 4 gives

$$
\begin{aligned}
\mid\{x & \left.\in \mathbf{R}^{n}: N_{\beta} u(x, \Psi(x))>2 \lambda\right\} \mid \\
& \leq C_{1} e^{-c_{2} / \varepsilon}\left|\left\{x \in \mathbf{R}^{n}: N_{\beta} u(x, \Psi(x))>\lambda\right\}\right| .
\end{aligned}
$$

Likewise, Theorem 3 combined with Lemma 5 gives

$$
\left.\mid x \in \mathbf{R}: N_{\beta} u(x, \Psi(x))>2 \lambda\right\}\left|\leq C_{1} e^{-c_{2} / \varepsilon^{2}}\right|\left\{x \in \mathbf{R}: N_{\beta} u(x, \Psi(x))>\lambda\right\} \mid .
$$

Note that in (5.5) the constant $L$ has been absorbed into the constant $c_{2}$.

We can then complete the proofs of Theorems 5 and 6 in the same fashion as Theorem 4. We just complete Theorem 6; the completion of Theorem 5 is similar. We have,

$$
\begin{aligned}
\sigma\{P & \left.\leq \partial D: N_{\beta} u(P)>2 \lambda, A_{\alpha} u(P)<\varepsilon \lambda\right\}=\sigma\left\{P \in E^{c}: N_{\beta} u(P)>2 \lambda\right\} \\
& \leq C\left|\left\{x \in \mathbf{R}: N_{\beta} u(x, \Psi(x))>2 \lambda\right\}\right| \\
& \leq c_{1} e^{-c_{2} / \varepsilon^{2}}\left|\left\{x \in \mathbf{R}: N_{\beta} u(x, \Psi(x))>\lambda\right\}\right| \\
& \leq c_{1} e^{-c_{2} / \varepsilon^{2}}\left|\left\{x \in \mathbf{R}: N_{\beta} u(x, \phi(x))>\lambda\right\}\right| \\
& \leq c_{1} e^{-c_{2} / \varepsilon^{2}} \sigma\left\{P \in \partial D: N_{\beta} u(P)>\lambda\right\} .
\end{aligned}
$$

\section{SOME APPLICATIONS AND REMARKS}

Theorems 1-6 also hold on starlike Lipschitz domains. The proofs are the same but slightly more technical since the boundary of such domains is covered by a finite number of coordinate charts rather than just one as in Theorems 1 and 6. Note that in this case we need a normalization like $u\left(P^{*}\right)=0$ where $P^{*}$ is the starcenter of the domain. This is because $A u$ (unlike $N u$ ) is unchanged if we alter $u$ by a constant. In Theorems 5 and 6 such a normalization is not needed since the inequalities are trivial if $\sigma\left\{N_{\beta} u(P)>\lambda\right\}=\infty$.

Suppose $\mu$ is a measure on $\partial D$ which satisfies an $A^{\infty}$ condition with respect to $\sigma$; that is, (2.1) remains valid with $\mu$ replacing $\sigma$. It is then immediate that 
Theorems 1, 2, 3 are true with $\mu$ replacing $\sigma$. Theorems 4, 5, and 6 remain valid with $\mu$ replacing $\sigma$ but this is not quite as evident. To see this, note that Lemmas 4 and 5 remain true if we consider a function $f$ on the boundary of a Lipschitz domain and use $\mu$ instead of Lebesgue measure as in these lemmas. Define a measure $\hat{\mu}$ on $\partial W$ by $\hat{\mu}(E)=\mu(p E)$ where $p E$ is the projection of $E$ onto $\partial W$. Then $\hat{\mu}$ satisfies an $A^{\infty}$ condition with respect to $\sigma$ on $\partial W$ and the constants which appear in condition (2.1) for $\hat{\mu}$ and $\sigma$ depend only on the constants for $\mu$ and $\sigma$ in (2.1) and the Lipschitz constant of $D$. In the case of Theorem 4, say, this allows one to replace $(5.1)$ by

$$
\hat{\mu}\left\{P \in \partial W: A_{\beta} u(P)>2 \lambda\right\} \leq C_{1} e^{-c_{2} / \varepsilon^{2}} \hat{\mu}\left\{P \in \partial W: A_{\beta} u(P)>\lambda\right\} .
$$

The proof then proceeds as before.

It is also quite likely that Theorems 4 and 5 remain valid if instead of considering harmonic functions $u$ on $D$ we consider functions $u$ which are solutions of $L u=0$ where $L$ is a uniformly elliptic operator with bounded measurable coefficients and we replace $\sigma$ by $L$ harmonic measure. Estimates for harmonic measure and Green's functions found in Dahlberg [7] and Hunt and Wheeden [12] have analogues in the elliptic case. (See Dahlberg, Jerison, Kenig [8].) However, the authors have not verified all the details of this.

Theorems 4, 5 and 6 also give estimates between the $L^{p}$ norms of $A u$ and $N u$ on $\partial D$. More precisely, we have,

Theorem 7. Let $\alpha>\beta$ and let $u$ be harmonic on an unbounded Lipschitz domain $D$ in $\mathbf{R}^{n+1}$. Then for $0<p<\infty$ there exists constants $c_{p}$ and $C_{p}$ such that

(i) $\left\|A_{\beta} u\right\|_{L^{p}(d \sigma)} \leq c_{p}\left\|N_{\alpha} u\right\|_{L^{p}(d \sigma)}$,

(ii) $\left\|N_{\beta} u\right\|_{L^{p}(d \sigma)} \leq C_{p}\left\|A_{\alpha} u\right\|_{L^{p}(d \sigma)}$.

If $n=1$ and $\alpha$ and $\beta$ are as in the statement of Theorem 6 , then there exists a constant $C_{p}^{\prime}$ such that

(iii) $\left\|N_{\beta} u\right\|_{L^{p}(d \sigma)} \leq C_{p}^{\prime}\left\|A_{\alpha} u\right\|_{L^{p}(d \sigma)}$.

Furthermore $c_{p}=O(\sqrt{p}), C_{p}=O(p)$ and $C_{p}^{\prime}=O(\sqrt{p})$ as $p \rightarrow \infty$ and $\sqrt{p}$ is the best possible order for $c_{p}, C_{p}^{\prime}$.

Parts (i) and (ii) of the theorem are already contained in [7]. In (iii), even for the case when $D=\mathbf{R}_{+}^{2}$, the behavior of the constant $C_{p}^{\prime}$ seems to be sharper than in any previous theorem known to the authors, all of which give at best, constants of order $p$. As the proof below shows the terms $e^{-c_{2} / \varepsilon^{2}}$ in Theorems 4 and 6 are absolutely essential to obtain $O(\sqrt{p})$ in (i) and (iii). Even the term $e^{-c_{2} / \varepsilon}$ in Theorem 5 would not enable us to obtain this order.

Proof. We assume that the norms of the functions in the right-hand sides of (i), (ii), (iii) are all finite. To obtain (i) we integrate the good- $\lambda$ inequality given by Theorem 4 . After rearranging we have

$$
\sigma\left\{A_{\beta} u>K \lambda\right\} \leq c_{1} e^{-c_{2} / \varepsilon^{2}} \sigma\left\{A_{\beta} u>\lambda\right\}+\sigma\left\{N_{\alpha} u>\varepsilon \lambda\right\} \text {. }
$$


Multiplying through by $p \lambda^{p-1}$ and integrating from 0 to $\infty$ gives

$$
\frac{1}{K^{p}}\left\|A_{\beta} u\right\|_{p}^{p} \leq c_{1} e^{-c_{2} / \varepsilon^{2}}\left\|A_{\beta} u\right\|_{p}^{p}+\frac{1}{\varepsilon^{p}}\left\|N_{\alpha} u\right\|_{p}^{p} .
$$

Therefore,

$$
\left(\frac{1}{K^{p}}-c_{1} e^{-c_{2} / \varepsilon^{2}}\right)\left\|A_{\beta} u\right\|_{p}^{p} \leq \frac{1}{\varepsilon^{p}}\left\|N_{\alpha} u\right\|_{p}^{p} .
$$

Choose $\varepsilon$ so that $1 / K^{p}-c_{1} e^{-c_{2} / \varepsilon^{2}}=1 / K^{p+1}$. Then $c_{2} / \varepsilon^{2}=(p+1) \log K+\log c_{1}$. So for $p$ large $1 / \varepsilon \leq c \sqrt{p}$. Therefore $c_{p}=O(\sqrt{p})$ as $p \rightarrow \infty$. The proofs of (ii) and (iii) follow from Theorems 5 and 6 in the same way.

We now show that for the constants $C_{p}$ and $C_{p}^{\prime}, O(\sqrt{p})$ is the best possible order as $p \rightarrow \infty$. To see this, consider $\mathbf{R}_{+}^{2}$ and a real valued function $f \in$ $L^{p}(\mathbf{R})$. Let $u$ be the harmonic extension of $f$ to $\mathbf{R}_{+}^{2}$ and let $v$ be the harmonic conjugate of $u$. Let $H f$ denote the Hilbert transform of $f$. Choose $\alpha<1<$ $\gamma<\beta$. Then

$$
\begin{aligned}
\|H f\|_{p} & =\|v\|_{p} \leq\left\|N_{\alpha} v\right\|_{p} \leq C_{p}^{\prime}\left\|A_{\gamma} v\right\|_{p}=C_{p}^{\prime}\left\|A_{\gamma} u\right\|_{p} \\
& \leq c_{p} C_{p}^{\prime}\left\|N_{\beta} u\right\|_{p} \leq C c_{p} C_{p}^{\prime}\|f\|_{p} .
\end{aligned}
$$

Therefore, $\|H f\|_{p} \leq C_{p}^{\prime \prime}\|f\|_{p}$ where $C_{p}^{\prime \prime}=O(p)$ as $p \rightarrow \infty$ and this is known to be the best possible order (Pichorides [17]).

Theorem 7 also remains valid on bounded starlike Lipschitz domains. In particular, if we consider the unit disc in $\mathbf{R}^{2}$, we get $\|f\|_{p} \leq C_{p}^{\prime}\left\|A_{\alpha}(f)\right\|_{p}$ with $C_{p}^{\prime} \leq C \sqrt{p}$ for $p \geq 2$. With the help of the central limit theorem for lacunary series one can show that the constant $C_{p}^{\prime}$ is of the best possible order (Moore [15, p. 20]). Here, in fact, we can obtain more information on this constant. Namely,

$$
\frac{1}{\sqrt{\alpha}} \frac{\sqrt{2}}{(2 \sqrt{\pi})^{1 / p}}\left(\Gamma\left(\frac{p+1}{2}\right)\right)^{1 / p} \leq C_{p}^{\prime}
$$

and by Stirling's formula we have that

$$
\lim _{p \rightarrow \infty} \frac{C_{p}^{\prime}}{\sqrt{p}} \geq \frac{1}{\sqrt{\alpha e}} .
$$

In (ii), $C_{p}=O(p)$; this is probably not the best possible order. It seems that it should be that $C_{p}=O(\sqrt{p})$, this would follow if one could obtain a higher dimensional version of Theorem 3 .

Theorems 4, 5, 6 also allow us to estimate the $L^{p}$ norms of the ratios $N u / A u$ and $A u / N u$. In fact, this was the motivation in [10] for improving the constants in the good- $\lambda$ inequalities. The theorem which follows is a generalization of a theorem of R. Fefferman, Gundy, Silverstein and Stein [10], and a theorem of Murai and Uchiyama [16]. 
Theorem 8. Let $\alpha>\beta$ and let $u$ be a harmonic function on an unbounded Lipschitz domain $D$. Then for every $p>0$,

$$
\begin{aligned}
& \int_{\partial D} \exp \left(c_{1} \frac{A_{\beta}^{2} u}{N_{\alpha}^{2} u}\right)\left(A_{\beta} u\right)^{p} d \sigma \leq c_{2} \int_{\partial D}\left(A_{\beta} u\right)^{p} d \sigma \\
& \int_{\partial D} \exp \left(c_{3} \frac{N_{\beta} u}{A_{\alpha} u}\right)\left(N_{\beta} u\right)^{p} d \sigma \leq c_{4} \int_{\partial D}\left(N_{\beta} u\right)^{p} d \sigma .
\end{aligned}
$$

If $D \subseteq \mathbf{R}^{2}$ and $D$ and $\alpha, \beta$ are as in Theorem 6 , then

$$
\int_{\partial D} \exp \left(c_{5} \frac{N_{\beta}^{2} u}{A_{\alpha}^{2} u}\right)\left(N_{\beta} u\right)^{p} d \sigma \leq c_{6} \int_{\partial D}\left(N_{\beta} u\right)^{p} d \sigma .
$$

Here $c_{1}, c_{3}, c_{5}$ depend only on $\alpha, \beta$ and the Lipschitz constant of $D$. The constants $c_{2}, c_{4}, c_{6}$ depend also on these parameters as well as $p$.

Theorem 8 follows from Theorems 4, 5, 6 in the same way as is shown in [10 or 16]. As before, part (iii) is new even when $D=\mathbf{R}_{+}^{2}$.

\section{LAWS OF THE ITERATED LOGARITHM FOR $N$ AND $A$}

Consider a function $u$ which is harmonic on $\mathbf{R}_{+}^{n+1}$ and let $\alpha>\beta$. We now give a direct comparison of the growth of $A_{\beta} u(x, y)$ and $N_{\alpha} u(x, y)$ as $y \rightarrow 0$.

Theorem 9. Suppose there exists a point $\left(x_{0}, y_{0}\right) \in \overline{\mathbf{R}_{+}^{n+1}}$ at which $A_{\alpha} u\left(x_{0}, y_{0}\right)<$ $\infty$. Then

$$
\limsup _{y \rightarrow 0} \frac{A_{\beta} u(x, y)}{N_{\alpha} u(x, y) \sqrt{\log \log N_{\alpha} u(x, y)}} \leq C
$$

almost everywhere on the set $\left\{x \in \mathbf{R}^{n}: N_{\alpha} u(x, 0)=\infty\right\}$. Here $C$ is a constant depending only on $\alpha, \beta$ and $n$.

Theorem 9 should be compared with the following result of I. Klemes and the authors in [1]. Suppose $u$ is harmonic in $\mathbf{R}_{+}^{2}$ and suppose $\alpha>\beta$ and $\alpha>1$. Then we have

Theorem 10 [1]. Suppose there exists a point $\left(x_{0}, y_{0}\right) \in \overline{\mathbf{R}_{+}^{2}}$ at which $N_{\alpha} u\left(x_{0}, y_{0}\right)$ $<\infty$. Then

$$
\limsup _{y \rightarrow 0} \frac{N_{\beta} u(x, y)}{A_{\alpha} u(x, y) \sqrt{\log \log A_{\alpha} u(x, y)}} \leq C
$$

almost everywhere on the set $\left\{x \in \mathbf{R}: A_{\alpha} u(x, 0)=\infty\right\}$. Here $C$ depends only on $\alpha$ and $\beta$.

Before proving Theorem 9 we make some remarks. The theorem will follow from Theorem 4. In a similar fashion Theorem 10 can be proved from Theorem 6. In [1], Theorem 10 was proved more directly and the proof avoids the results of Dahlberg [7] and Hunt and Wheeden [12] which were required to prove 
Theorem 3. Theorem 5 also leads to a law of the iterated logarithm but it is much weaker and we will not give its proof.

Let $X_{t}$ be a continuous martingale with square function $\langle X\rangle_{t}$. Define $X_{t}^{*}=$ $\sup _{s \leq t}\left|X_{s}\right|$. Then the law of the iterated logarithm for continuous martingales (see Durrett [9]) states that

$$
\limsup _{t \rightarrow \infty} \frac{X_{t}^{*}}{\sqrt{2\langle X\rangle_{t} \log \log \langle X\rangle_{t}}}=1
$$

a.e. on the set $\left\{\langle X\rangle_{\infty}=\infty\right\}$. A basic philosophy is that $X_{\infty}^{*}$ and $\langle X\rangle_{\infty}^{1 / 2}$ behave like $N u$ and $A u$. This is the inspiration behind Theorem 10. For the proof of Theorem 10 and applications to other laws of the iterated logarithm in the analysis setting see [1 and 2].

Proof of Theorem 9. We can assume that $x_{0}=0$ and that $A_{\alpha} u\left(0, y_{0}\right) \leq 1$. It suffices to show that for each positive integer $M,(7.1)$ holds on $|x| \leq M$. Fix $M$. We may assume that there exists a point $(x, y)$ with $|x| \leq M, y>0$ such that $N_{\alpha} u(x, y)<\infty$. This is because the condition that $A_{\alpha} u\left(0, y_{0}\right)<\infty$ insures that $A_{\beta} u(x, y)<\infty$ for all such $(x, y)$ and if $N_{\alpha} u(x, y)=\infty$ for all such $(x, y)$ then $(7.1)$ is trivially true. We can then define functions $\phi_{k}: \mathbf{R}^{n} \rightarrow$ $\mathbf{R}$ as follows. For $|x| \leq M$ set $\phi_{k}(x)=\inf \left\{y>0: N_{\alpha} u(x, y) \leq 2^{k}\right\}$. Pick $\gamma$ so that $\alpha>\gamma>\beta$. Then set $W_{k}=\bigcup_{\{x:|x| \leq M\}} \Gamma_{\gamma}\left(x, \phi_{k}(x)\right)$. The region $W_{k}$ has the property that if $P \in \partial W_{k}$ then $\Gamma_{\gamma}(P) \subseteq W_{k}$; consequently, $\partial W_{k}$ is the graph of a Lipschitz function whose constant depends only on $\alpha$. For $|x| \leq M, \partial W_{k}$ is the graph of $\phi_{k}$ so we can extend $\phi_{k}$ to $\mathbf{R}^{n}$ by requiring that the graph of $\phi_{k}$ is $\partial W_{k}$. We note that $\phi_{k} \geq \phi_{k+1}$ for all $k$. Since $\alpha>\gamma>\beta$, then there exists a constant $M^{\prime}$ depending only on $y_{0}, M, \alpha$ and $\beta$ such that if $|x|>M^{\prime}$, then $\Gamma_{\beta}\left(x, \phi_{k}(x)\right) \subseteq \Gamma_{\alpha}\left(0, y_{0}\right)$ for any $k$. Then a slight variation of Lemma 11 shows that if $|x|>M^{\prime}, A_{\beta} u\left(x, \phi_{k}(x)\right) \leq L$ where $L$ depends only on $\beta$ and $\gamma$.

Consider any $\lambda>L$. Then by Theorem 4 , for every $j$ we have

$$
\begin{aligned}
\sigma\{P & \left.\in \partial W_{j}: A_{\beta} u(P)>K \lambda, N_{\gamma} u(P) \leq \varepsilon \lambda\right\} \\
& \leq c_{1} e^{-c_{2} / \varepsilon^{2}} \sigma\left\{P \in \partial W_{j}: A_{\beta} u(P)>L\right\} .
\end{aligned}
$$

Apply this with

$$
\lambda=2^{j} \sqrt{\frac{2}{c_{2}} \log \log 2^{j}} \text { and } \varepsilon=\left(\frac{2}{c_{2}} \log \log 2^{j}\right)^{-1 / 2},
$$

and note that for this $\varepsilon, \lambda, N_{\gamma} u(P) \leq \varepsilon \lambda$ for every $P \in \partial W_{j}$. We obtain

$$
\begin{aligned}
& \sigma\{P\left.\in \partial W_{j}: A_{\beta} u(P)>K 2^{j} \sqrt{\frac{2}{c_{2}} \log \log 2^{j}}\right\} \\
& \leq c_{1}(j \log 2)^{-2} \sigma\left\{P \in \partial W_{j}: A_{\beta} u(P)>L\right\} .
\end{aligned}
$$


Set

$$
E_{j}=\left\{x:|x| \leq M, A_{\beta} u\left(x, \phi_{j}(x)\right)>K 2^{j} \sqrt{\frac{2}{c_{2}} \log \log 2^{j}}\right\} .
$$

Since $\phi_{j}$ is Lipschitz and $\sigma\left\{P \in \partial W_{j}: A_{\beta} u(P)>L\right\} \leq C$ where $C$ is a constant independent of $j$, then $\sum_{j=1}^{\infty}\left|E_{j}\right| \leq C \sum_{j=1}^{\infty} 1 / j^{2}<\infty$. By the BorelCantelli lemma, almost all $x$ are in only a finite number of the sets $E_{j}$. That is, there exists a $j_{0}$ (depending on $x$ ) such that for all $j \geq j_{0}, x \notin E_{j}$. Consider such an $x$ for which we also have $N_{\alpha} u(x, 0)=\infty$ and say $\phi_{j}(x) \searrow s \geq 0$. If $s>0$ then for all $y<s, N_{\alpha} u(x, y)=\infty$ and so (7.1) is trivially true for this $x$. If $s=0$, consider any $y>0$ such that $y \leq \phi_{j_{0}}(x)$. Then there exists a $j \geq j_{0}$ such that $\phi_{j+1}(x)<y \leq \phi_{j}(x)$ and so $2^{j} \leq N_{\alpha} u(x, y)<2^{j+1}$. Using Lemma 11 we have

$$
\begin{aligned}
A_{\beta} u(x, y) & \leq C A_{\beta} u\left(x, \phi_{j+1}(x)\right)<C K 2^{j+1} \sqrt{\frac{2}{c_{2}} \log \log 2^{j+1}} \\
& \leq C N_{\alpha} u(x, y) \sqrt{\log \log N_{\alpha} u(x, y)}
\end{aligned}
$$

finishing the proof of Theorem 9.

The good- $\lambda$ inequalities above combined with the techniques of Burkholder and Gundy [4] can be used to show the following analogue for Lipschitz domains of the Calderón-Stein result for $\mathbf{R}^{n}$.

Theorem 11. Let $D$ be a Lipschitz domain (any dimension) and let $u$ be harmonic in $D$. Then the set where the area function is finite equals almost everywhere with respect to surface measure the set where the nontangential maximal function of $u$ is finite.

This result in fact holds for the more general NTA domains as was shown in D. Jerison and C. Kenig [13]. The theorem below, whose proof is the same as the proof of Theorem 9, gives more information on the behavior of these functions on the complement of the sets of Theorem 11.

Theorem 12. Let $\alpha>\beta$ and let $u$ be a harmonic function on the Lipschitz domain $D=\left\{(x, y): x \in \mathbf{R}^{n}, y>\phi(x)\right\}$. Suppose there exists a point $\left(x_{0}, y_{0}\right)$ with $y_{0} \geq 0$ such that $A_{\alpha} u\left(x_{0}, \phi\left(x_{0}\right)+y_{0}\right)<\infty$. Then

$$
\limsup _{y \rightarrow 0} \frac{A_{\beta} u(P+(0, y))}{N_{\alpha} u(P+(0, y)) \sqrt{\log \log N_{\alpha} u(P+(0, y))}} \leq C
$$

$\sigma$ almost everywhere on $\left\{P \in \partial D: N_{\alpha} u(P)=\infty\right\}$. Here $C$ depends on $\alpha, \beta$, $n$, and the Lipschitz constant of $D$.

\section{REFERENCES}

1. R. Bañuelos, I. Klemes and C. N. Moore, An analogue of Kolmogorov's law of the iterated logarithm for harmonic functions, Duke Math. J. 57 (1988), 37-68.

2. R. Bañuelos and C. N. Moore, Some results in analysis related to the law of the iterated logarithm, Proc. Special Year in Analysis, Univ. of Illinois (to appear). 
3. D. L. Burkholder, Exit times of Brownian motion, harmonic majorization and Hardy spaces. Adv. in Math. 26 (1977), 182-205.

4. D. L. Burkholder and R. F. Gundy, Distribution function inequalities for the area integral, Studia Math. 44 (1972), 527-544.

5. S.-Y. A. Chang, J. M. Wilson and T. H. Wolff, Some weighted norm inequalities involving the Schrödinger operators, Comment. Math. Helv. 60 (1985), 217-246.

6. B. E. J. Dahlberg, Estimates of harmonic measure, Arch. Rational Mech. Anal. 65 (1977), 272-288.

7. $\ldots$, Weighted norm inequalities for the Lusin area integral and the nontangential maximal functions for functions harmonic in a Lipschitz domain, Studia Math. 47 (1980), 297-314.

8. B. E. J. Dahlberg, D. S. Jerison and C. E. Kenig, Area integral estimates for elliptic differential operators with non-smooth coefficients, Ark. Math. 22 (1984), 97-108.

9. R. Durrett, Brownian motion and martingales in analysis, Wadsworth, Belmont, Calif., 1984.

10. R. Fefferman, R. F. Gundy, M. Silverstein and E. M. Stein, Inequalities for ratios of functionals of harmonic functions, Proc. Nat. Acad. Sci. U.S.A. 79 (1982), 7958-7960.

11. J. Garnett, Bounded analytic functions, Academic Press, New York, 1980.

12. R. A. Hunt and R. L. Wheeden, On the boundary values of harmonic functions, Trans. Amer. Math. Soc. 132 (1968), 307-322.

13. D. S. Jerison and C. E. Kenig, Boundary behavior of harmonic functions in non-tangentially accessible domains, Adv. in Math. 46 (1982), 80-147.

14. C. E. Kenig, Weighted $H^{p}$ spaces on Lipschitz domains, Amer. J. Math. 102 (1980), 129-163.

15. C. N. Moore, Some applications of Cauchy integrals on curves, Ph. D. Thesis, Univ. of California at Los Angeles, 1986.

16. T. Murai and A. Uchiyama, Good- $\lambda$ inequalities for the area integral and the nontangential maximal function, Studia Math. 83 (1986), 251-262.

17. S. K. Pichorides, On the best constants in the theorems of M. Riesz, Zygmund and Kolmogorov, Studia Math. 44 (1972), 165-179.

18. E. M. Stein, Singular integrals and differentiability properties of functions, Princeton Univ. Press, Princeton, N. J., 1970.

19. J.-O. Strömberg, Bounded mean oscillation with Orlicz norms and duality of Hardy spaces, Indiana Univ. Math. J. 28 (1979), 511-544.

Department of Mathematics, University of Illinois, Urbana, Illinois 61801

Department of Mathematics, Washington University, St. Louis, Missouri (Current address of C. N. Moore)

Current address (Rodrigo Banuelos): Department of Mathematics, Purdue University, West Lafayette, Indiana 47907 